\title{
Human Detection Implementation on UAVs in Search and Rescue Operation
}

\author{
Muhammad Shahir Hakimy Salem, Fadhlan Hafizhelmi Kamaru Zaman*, and Nooritawati Md Tahir
}

\begin{abstract}
Through the years, natural disasters keep happening and becoming more frequent and severe. Disasters can cause loss of property as well as human life. In disasters that cause mass fatalities, search and rescue operations are indeed vital and need to be conducted urgently. Unmanned aerial vehicles (UAV) or drones are the new emerging technology that has the capability to be deployed regularly to aid in search and rescue. By using UAV technology, it can speed up the search and rescue process and speed-up victims' identification process before the body decomposes. Compared to conventional search and rescue operations, UAVs are more appropriate and economical due to their small-scale size of equipment. This paper presents reviews on the use of human detection based on UAV and technology in the scope of search and rescue operations for natural disasters.
\end{abstract}

Index Terms - Human Detection, Unmanned Aerial Vehicle (UAV), Natural Disaster.

\section{INTRODUCTION}

$I_{s}^{N}$ $\mathrm{N}$ the past decade, various disasters happened in the world such as earthquakes, floods, and Tsunami. The definition of disasters defined by the Malaysian National Security Council (MNSC) directive 20 (2003) states that "an emergency situation that will cause the loss of lives, damage property, and the environment, and hamper local, social, and economic activities" [1]. Figure 1 shows the statistic of natural disaster victims in 2019 versus the type of disaster around the world [2]. Around 1.5 million people were affected by earthquakes and 31 million by floods across the world. Malaysia relatively free from natural disasters, but affected by landslides, haze, flooding, and other man-made disasters. It is because Malaysia lies in a geographically stable region and this is caused by human activity like disrupting the natural balance by cutting trees, destroying hills, and unplanned urbanization, the natural disaster keeps happening frequently [3].

Once the disaster happens, search and rescue (SAR) teams play an important role in this situation. One major problem arises for the rescue team is in finding and identifying the victim's location process [4].

This manuscript is submitted on 3rd February 2021 and accepted on 15th March 2021. This work is supported by Ministry of Education (MOE) Malaysia under the grant 600-IRMI/TRGS 5/3 (001/2019)-1

Muhammad Shahir Hakimy Salem, Fadhlan Hafizhelmi Kamaru Zaman and Nooritawati Md Tahir are with the Faculty of Electrical Engineering, Universiti Teknologi MARA, 40450 Shah Alam, Selangor (fadhlan@uitm.edu.my)

1985-5389/C 2021 The Authors. Published by UiTM Press. This is an open access article under the CC BY-NC-ND license (http://creativecommons.org/ 70 licenses/by-nc-nd/4.0/).
The longer the time taken to find and identify the victim's location caused delay for the rescue team to reach the victims. The finding and identifying the victim location process affects the rescue teams in the rescue process. Limited time available to gather evidence and identify the body before decomposition begins, especially in tropical weather. Search and rescue operations consumed countless time and cost if carried out by boat and helicopter because helicopters need to fly from the base or an airfield to the disaster area. The estimation of deployment of helicopters in search and rescue situations was $\$ 10,000-\$ 15,000$ per hour [5]. Nowadays, the drone is one of the applications that has been chosen to find and identify the victim's location. A mission such as a search and a rescue or forest surveillance requires large camera coverage and thus making the drone a suitable technique to perform advanced tasks.

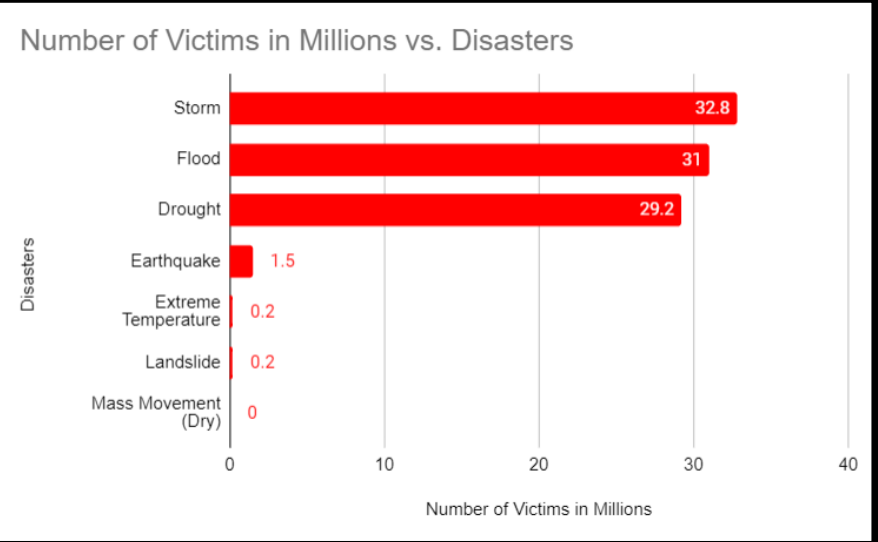

Fig. 1. Statistic of natural disaster victims in 2019 around the world [2].

Apart from the importance of choosing drone criteria, equipping the system and algorithm in the drone is also one of the important things that need to be considered. Recently, deep CNNs have been established as one of the most promising avenues of research in the computer vision area, with outstanding results in a series of visual recognition tasks, such as image classification [6], [7], face recognition [8], digit recognition [9], [10], pose estimation [11], object and pedestrian detection [12], [13], and content-based image retrieval [14], [15]. The increasing trend of human detection 
application in computer vision provides a remarkable insight into a search and rescue situation [16]. It also has the capability to run body detection algorithms and face identification algorithms to detect, localize, and identify human forms. The effectiveness of human detection and victim localization using image processing and drone technology should be more rapidly as compared to conventional manual search and rescue techniques [17]. Detecting humans in real-time situations is a challenging task. It is because of the lighting, environment, their variable appearance, and the wide range of poses that they can adopt.

\section{SEARCH AND RESCUE (SAR) OPERATION}

Through the years, natural disasters keep happening and becoming more frequent and severe. Numerous efforts have been made in order to handle the disaster and search and rescue (SAR) operation. There are many agencies that take place in search and rescue operation around the world such as National Association for Search and Rescue (NASAR) in the United States, Civil Air Search and Rescue Association (CASARA) in Canada, Aeronautical Rescue Coordination Centre (ARCC) in England, South African Maritime Safety Authority (SAMSA) in South Africa and Australian Maritime Safety Authority (AMSA) in Australia. In Malaysia, two main SAR units are Special Malaysia Disaster Assistance and Rescue Team (SMART) which reports to the National Security Council and Malaysian Fire and Rescue Department (FRDM) and Special Tactical Operation and Rescue Team of Malaysia (STORM) unit[18]. These units are also supported by another jungle expert team and aboriginal police unit. Through the year, the conventional search and rescue facilities that have been used are boat and helicopter with high cost and longer time operations. This search and rescue operation will affect the victim's identification process as shown in part three.

Normally, the fundamentals which will affect the search and rescue operation are divided into six-part that is an organization, management, leadership, strategy, tactics, and clue awareness. All these basic principles and theories will normally render successful searches. However, during search and rescue operations, there are several key actions and decisions that must be made by the search and rescue management personnel. The consideration that needs to be prioritized and expedited for all search and rescue responders is they need to prepare the plan early, identify all hazards in the area (think safety), manage all resources effectively and efficiently, make analysis and evaluation an ongoing process, and gather all the information that available and keep its records of all actions taken.

According to search and rescue standard operating procedures [19] , search management can be divided into threepart that is a pre-incident, incident, and post-incident.

\section{A. Pre-Incident}

a) Interagency coordination

Firstly, management needs to identify all the agency responders, lines of authority, comprehensive communication, and area of responsibilities and tasks.

\section{b) Logistic support}

This part considers as one of the vital parts that need to be a plan as best as can. It will affect the process of search in order to get supply especially search and safety equipment, researcher's requirement, and communication systems. Another special need is cost coverage for equipment replacement and repairs.

\section{c) Facility Disposition}

Some area needs to establish such as base camp, family area, media area, and staging area.

\section{B. Incident}

a) Rapid Response

In this part, rapid responses from the management need to be taken. The management needs to determine search urgency and establish confinement of area. Determine the last known position or location last seen. It will help to allocate the segmentation of the search area.

b) Area Management

In order to manage the area, some consideration needs to take place such as access control, implement facility plan and identifying the resources.

c) Incident Action Plan (IAP) Development IAP development considers the development and implements workable search areas. Places highly trained or skilled resources into high probability areas and evaluate ongoing information.

\section{d) Standard Operation Procedures}

Keep records of all the information such as worker compensation enrollment, maps, and charts, ICS forms, and searcher identification. All the ongoing search strategies and tactics need to follow the standard operating procedure. Other than that, management needs to prepare for searcher, family, and media briefing.

\section{Post-Incident}

\section{a) Final Briefing}

Recognize all the agencies and organization that takes place in search and rescue process. 


\section{b) Demobilization}

Clean up of command post and all the area that has been used during the search and rescue process.

\section{c) Evaluation}

Analyze all the plan and standard operating processes such as direction and control, training of personnel, and communication system.

\section{d) Reporting}

Write a search and rescue report form (written after-action).

\section{CONVENTIONAL DISASTER VICTIMS IDENTIFICATION (DVI)}

Identification of the victims after the disaster is a challenging task as well. The aim of the process is to accurately identify the victims of the disaster or incident before being claimed by their respective families [20]. The current DVI method consists of four phases: Scene Phase, Post-mortem Phase, Ante-mortem Phase, and Reconciliation Phase.

\section{A. Scene Phase}

After a mass disaster, in the first phase, the forensic team proceeds to the field and examines the victims as survive or otherwise. If dead, their corpse will be labeled and any identification is safely kept as evidence [21]. These labels should carry information such as examining the team, location found, and victim number. The area of disaster is usually divided into quadrants and teams are dispatched to each quadrant to execute this early stage of identification.

\section{B. Post-mortem Phase}

After the victims have been removed from the disasters, the second phase is to conduct the post-mortem examination. This second phase can include post-mortem identification, which can be carried out in conjunction with the first phase. An examination may include capturing fingerprints [22], teeth examination [23], body examination, any materials found on the body as well as collection of samples for DNA examination [24].

\section{Ante-mortem Phase}

The third phase includes ante-mortem data collection from others such as family members and medical records. This process can be lengthy as the interviewer will ask a series of predetermined questions to assist the identification process of victims. This will include questions about the description of the missing person, jewelry, clothing, and other personal items belong to the victims. Ante-mortem records will be used to verify victim identification by comparing it with Postmortem records [25]. Due to dangerous terrains, extreme weather, a wide disaster area, and a large number of victims involved, there is usually no room for victim identification and postmortem examination at the site of disaster.

\section{Reconciliation Phase}

Lastly, after the post-mortem and ante-mortem details have been conducted, a team of specialists will meet and commence to reconcile both sets of information to determine the identification of the deceased victims. Once the victim has been identified, the process of releasing the corpse to the family members can be done.

\section{UnMaNNEd AERIAL VeHIClE (UAV) APPliCATION}

The employment of robots as a support platform for management applications is becoming a trend in managing and accessing natural disasters. There are various types of robots that can help society and the nation in order to manage and access natural disasters. One of the robots used in the support platform is an unmanned vehicle (UV). An unmanned vehicle or un-crewed vehicle is a means of transportation without a person on board and it can either be controlled remotely or as an autonomous vehicle. In 2020 only, the world has experienced a few disasters such as Beirut Explosion [26], California Wildfires [27], and the Philippine Volcano eruption [28].

Drones have been widely used to capture images or views of the affected areas. Images and videos of drones surveying disaster areas are widely available on the online platform. However, there are few challenges that need to be overcome or to be improved to enhance drone capabilities in attending the situation. In daily tasks, UAV has been deployed in industrial areas for inspection process [29] - [34], surveying and mapping [35] [36], land monitoring, security and emergency response [37] - [39], stockpile management, and haul road [40] - [42]. As all know, drones come with a few embedded devices that are flight control, power circuit, transceiver, global positioning system, and a few sensors that can make them more useful and powerful. The high-performance drone will be used for difficult tasks and requires precision such as searching for flood victims or survivors [43], flood surveillance [44], forensic purpose [45] [46], and mapping in post-disaster volcano eruption [47].

According to [48], one of the important parts of search and rescue is maintaining communication over the disaster areas. Once a disaster occurs, public communication network may be unavailable in the remote area. Secondly, if it is available the network may be damaged or destroyed. So, in this situation, to extend the coverage on the affected area drone can be used temporarily as mobile access point. Multiple UAV is used in order to reach the target area the soonest possible while saving the maximum number of people. According to [49], using a layered search and rescue (LSAR) algorithm, it more efficient in searching for survivor in minimum time. This is because the LSAR algorithm focuses on the center of the disaster.

\section{DRONE TECHNOLOGY}

Detection is a process of determining the location on the image where certain objects are present, as well as classifying those objects. Nowadays, technology has grown rapidly, and it leads researchers to the production of various types of human detection such as acoustic sensors [50], infrared detectors [51], 
radar [52], and image recognition of human shapes [53]. There are also some studies that proposed to use a drone with image processing to aid body detection.

Nowadays, every human will definitely have at least 1 cellphone equipped with a fourth-generation (4G) Long Term Evoluation (LTE) [54]. In another way to localize victims that trap in disaster areas is using radio frequency $(\mathrm{RF})$.

Control system piloting the drone, software running in the drone and the connection between the two is an unmanned aerial system (UAS) that allowed the UAV to function [55]. Some researchers have made research in detecting human crowds using a convolution neural network (CNN) for drone flight safety. According to [56], by provided the crowd heat maps in the drone technology or system, it will semantically enhance the flight maps by defining no-fly zone.

Drone technology was very helpful in search and rescue process. Once victims have been detected, rescue team can monitor the affected areas before getting into it. Some drones equipped with object collision detection sensor and communication modules. UAVs with fully autonomous functional was very unique system but existing system may damage if UAV is damage-free during detection [57].

\section{A. UAVs Used in Search and Rescue (SAR) Operation}

The deployment of the drone in search and rescue has become a very popular topic. In addition to skilled searchers in search of victims, UAVs technology also may help to ensure success in the search or localize the victims in the disaster area. The drone has been used by the response team in the 2017 hurricane season [58]. The drone has been used to localize victims, access flood damage, and predict areas of future flood risk.

Due to the earthquake that occurred in 2015 in Nepal, it's killing over 7,500 people and destroy homes and buildings. The drone [59] has been used in managing the search and rescue operation, gather imagery, identifying the affected area, and assess the extent of the damage. All the data or information which has been picked up by a drone will be sent to rescue workers on the ground directly. It will help the rescue worker to go directly to the high potential victim's areas.

In Amherst Country, Virginia, the tornado has destroyed dozens of homes and injuring people. The drone has been used in order to help by providing aerial views by using a highresolution camera to locate injured persons and personal belongings of homes that had been destroyed [60]. Just like what happened in Leilani Estates, Hawaii, the drone has been used to identify the number of houses destroyed after the Kilauea Volcano [61] erupted and spewed lava that happens in May 2018.

In 2017, a drone has been used for the first time ever by Los Angeles Fire Department [62]. The drones mainly used to locate victims that trap in fire and access property damage caused by the fire. This drone technology used due to several accidents that happens between 1990 and 2017 which an average of 17 firefighters died per year during activities related to fighting wildfire. The manned aircraft flies at low altitudes and putting them at risk of low visibility, dangerous winds, and high temperature.

\section{B. Type of Drone}

The drone can be divided into three types: the air, ground, and underwater drone as shown in Figure 2. The drone is one of the intelligent inventions that are more efficient, cost-effective, and could reduce human error and ease manpower. According to [35], it proved that using UAVs has high accuracy, quicker (saves time), and easier to deploy. Drones can then be equipped with some intelligence to carry out some of the early search victims' locations. The drone also has three types of area of differences that is quadcopter, hexacopter, and octocopter as shown in table 1. According to [5], the quadcopter has a longer flying duration and great maneuverability.

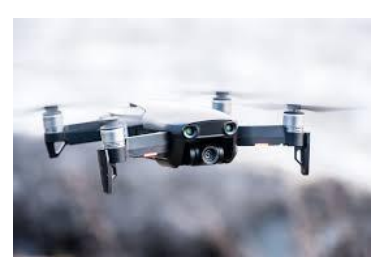

(a)

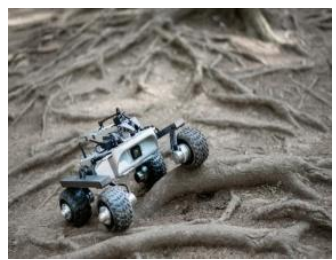

(b)

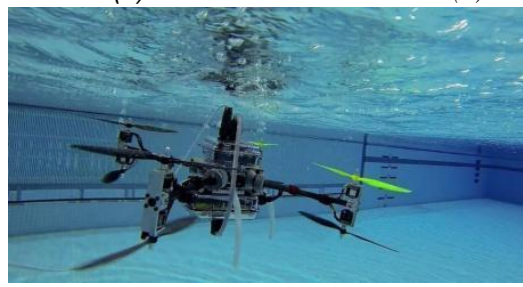

(c)

Fig. 2. Type of drones (a) air drone (b) ground drone and (c) underwater drone.

TABLE 1

COMPARATIVE STUDY ON QUADCOPTER, HEXACOPTER, AND OCTOCOPTER

\begin{tabular}{|c|c|c|c|}
\hline $\begin{array}{c}\text { Area of } \\
\text { Difference }\end{array}$ & Quadcopter & Hexacopter & Octocopter \\
\hline $\begin{array}{c}\text { Number of } \\
\text { Propellers }\end{array}$ & 4 & 6 & 8 \\
\hline Advantages & $\begin{array}{c}\text { Cheap to build } \\
\text { and repair. }\end{array}$ & $\begin{array}{c}\text { Great power } \\
\text { and speed. }\end{array}$ & $\begin{array}{c}\text { Very fast, agile } \\
\text { and stable. }\end{array}$ \\
\hline Disadvantages & $\begin{array}{c}\text { Less powerful } \\
\text { engine }\end{array}$ & $\begin{array}{c}\text { High price, } \\
\text { Larger in size }\end{array}$ & Very expensive \\
\hline
\end{tabular}

\section{Computer Vision}

Drone technology has advanced to a stage that the most advanced ones are equipped with autonomous flight capabilities, computer vision, and on-board artificial intelligence (AI) technology and processing. Over the last few years, deep learning conquered the computer vision field by providing a good solution to a difficult problem. Deep learning has a lot of architecture that are convolutional neural networks $(\mathrm{CNN})$, recurrent neural networks (RNN), generative adversarial neural networks (GAN), ResNet, etc. Each type of deep-learning architecture mitigates a different type of problem. CNN have been established as one of the most 
promising avenues of research in the computer vision area, providing outstanding results in a series of vision recognition tasks, such as image classification [63], [64], face recognition [65], digit recognition [66],[67], pose estimation [68], object and pedestrian detection [69], [70], and content-based image retrieval [71], [72].

\section{Impact of UAV Application toward Search and Rescue Operation}

Most researchers that have conducted research on UAVs led success in dealing with the problems that occur. Day by day, the earth is getting worse and inviting natural disasters. With the increasing number of natural disasters recently, the development and use of drones in search and rescues operation become more important. The main advantage of using drones in search and rescue operations is all about time. With this technology, reaching the disaster sites will be more rapid and reduced the searching time of the site and can pursuit immediately for victims or survivals. The drone can be deployed into the catastrophe sites hurriedly because of less risk as compared to human life [73]. Other than that, drones can also give awareness in a large area speedily, reducing time and the number of searchers that need to locate and rescue the victims or survivors. Besides that, it greatly reduces search expenses and risk in rescue missions. The drone has been designed to provide real-time data and imaging, day or night, harsh conditions without danger to work for and cost-efficient too [74].

\section{CONCLUSION}

In conclusion, the uses of UAVs in search and rescue operations during natural disasters are presented. Technology advancement and impact of use of drones in real situations are elaborated and discussed. However, there are still not many researches done to aid the forensic management team in locating and identifying deceased victims in remote areas. Hence, more research is required to utilise drones in natural disaster management and determine suitable processes to be carried out by drones, given the exact capabilities and battery life to be employed by the drones.

\section{ACKNOWLEDGMENT}

The authors would like to thank the Ministry of Education (MOE) Malaysia for providing the grant 600-IRMI/TRGS 5/3 (001/2019)-1, and the Research Management Centre (RMC) of Universiti Teknologi MARA for supporting this research work.

\section{REFERENCES}

[1] Shah, Syed \& Mustaffa, Zahiraniza \& Wan Yusof, Khamaruzaman. "Disasters Worldwide and Floods in the Malaysian Region: A Brief Review," in Indian Journal of Science and Technology, 2017.

[2] Statista Research Department: "Natural disaster victims worldwide by type of catastrophe 2019", Dec 1, 2020.

[3] Chan, Ngai Weng. (2015). Impacts of Disasters and Disaster Risk Management in Malaysia: The Case of Floods. 10.1007/978-4-43155022-8.
[4] Uddin, Zia \& Islam, Mojaharul. (2016). Search and rescue system for alive human detection by semi-autonomous mobile rescue robot. 1-5. 10.1109/ICISET.2016.7856489.

[5] Hashim, Ahmad \& Tamizi, Mohamad. (2018). Development of Drone for Search and Rescue Operation in Malaysia Flood Disaster. International Journal of Engineering \& Technology. 7. 9. 10.14419/ijet.v7i3.7.16195.

[6] A. Krizhevsky, I. Sutskever, and G. E. Hinton, "Imagenet classification with deep convolutional neural networks," in Advances in neural information processing systems, 2012, pp. 1097-1105.

[7] C. Szegedy, W. Liu, Y. Jia, P. Sermanet, S. Reed, D. Anguelov, D. Erhan, V. Vanhoucke, and A. Rabinovich, "Going deeper with convolutions," in Proceedings of the IEEE Conference on Computer Vision and Pattern Recognition, 2015, pp. 1-9.

[8] Y. Taigman, M. Yang, M. Ranzato, and L. Wolf, "Deepface: Closing the gap to human-level performance in face verification," in Proceedings of the IEEE Conference on Computer Vision and Pattern Recognition. IEEE, 2014, pp. 1701-1708

[9] D. Ciresan, U. Meier, and J. Schmidhuber, "Multi-column deep neural networks for image classification," in Computer Vision and Pattern Recognition (CVPR), 2012 IEEE Conference on. IEEE, 2012, pp. 36423649.

[10] Y. LeCun, L. Jackel, L. Bottou, C. Cortes, J. S. Denker, H. Drucker, I. Guyon, U. Muller, E. Sackinger, P. Simard et al., "Learning algorithms for classification: A comparison on handwritten digit recognition," Neural networks: the statistical mechanics perspective, vol. 261, p. 276, 1995.

[11] A. Toshev and C. Szegedy, "Deeppose: Human pose estimation via deep neural networks," in Proceedings of the IEEE Conference on Computer Vision and Pattern Recognition, 2014, pp. 1653-1660.

[12] R. Girshick, J. Donahue, T. Darrell, and J. Malik, "Rich feature hierarchies for accurate object detection and semantic segmentation," in Proceedings of the IEEE conference on computer vision and pattern recognition, 2014, pp. 580-587.

[13] P. Sermanet, K. Kavukcuoglu, S. Chintala, and Y. LeCun, "Pedestrian detection with unsupervised multi-stage feature learning," in Computer Vision and Pattern Recognition (CVPR), 2013 IEEE Conference on. IEEE, 2013, pp. 3626-3633.

[14] A. Babenko, A. Slesarev, A. Chigorin, and V. Lempitsky, "Neural codes for image retrieval," in Computer Vision-ECCV 2014. Springer, 2014, pp. 584-599.

[15] J. Wan, D. Wang, S. C. H. Hoi, P. Wu, J. Zhu, Y. Zhang, and J. Li, "Deep learning for content-based image retrieval: A comprehensive study," in Proceedings of the ACM International Conference on Multimedia. ACM, 2014, pp. 157-166

[16] S. Yong and Y. Yeong, "Human Object Detection in Forest with Deep Learning based on Drone's Vision," 2018 4th International Conference on Computer and Information Sciences (ICCOINS), Kuala Lumpur, 2018, pp. 1-5, doi: 10.1109/ICCOINS.2018.8510564.

[17] Duc Thanh Nguyen, Wanqing Li, Philip O. Ogunbona, "Human detection from images and videos: A survey", Pattern Recognition,Volume 51,2016,Pages 148-175,ISSN 0031-3203.

[18] Półka, Marzena \& Ptak, Szymon \& Kuziora, Łukasz. (2017). The Use of UAVs for Search and Rescue Operations. Procedia Engineering. 192. 748-752. 10.1016/j.proeng.2017.06.129.

[19] Dem, July, 1998, "Search and Rescue Standard Operating Procedure", http://jcsda.com/docs/sar_mgt.pdf.

[20] Harald J. Meyer, Nantarika Chansue, Fabio Monticelli, "Implantation of radio frequency identification device (RFID) microchip in disaster victim identification (DVI), "Forensic Science International, Volume 157, Issues 2-3, 2006, Pages 168-171, ISSN 0379-0738, https://doi.org/10.1016/j.forsciint.2005.10.001.

[21] Smith, Adrian, "Aviation, Space, and Environmental Medicine", Volume 74, Number 11, November 2003, pp. 1198-1200(3)

[22] K. Panetta, S. Rajeev, K. M. S. Kamath and S. S. Agaian, "Unrolling PostMortem 3D Fingerprints Using Mosaicking Pressure Simulation Technique," in IEEE Access, vol. 7, pp. 88174-88185, 2019.

[23] Elisa Nguyen, Edel Doyle, "Dental Post-mortem Computed Tomography for Disaster Victim Identification: A literature review", Journal of Forensic Radiology and Imaging, Volume 13, 2018, Pages 5-11, ISSN 2212-4780

[24] D. Hartman, O. Drummer, C. Eckhoff, J.W. Scheffer, P. Stringer, "The contribution of DNA to the disaster victim identification (DVI) effort", Forensic Science International, Volume 205, Issues 1-3, 2011, Pages 5258, ISSN 0379-0738.

[25] I. Amundsen, A.M.L. Øiestad, D. Ekeberg, L. Kristoffersen, "Quantitative determination of fifteen basic pharmaceuticals in ante- and 
post-mortem whole blood by high $\mathrm{pH}$ mobile phase reversed phase ultra high performance liquid chromatography-tandem mass spectrometry", Journal of Chromatography B, Volume 927, 2013, Pages 112-123, ISSN 1570-0232.

[26] H. Ben and A.-H. Maria, "Deadly Explosions Shatter Beirut, Lebanon," The New York Times, Aug. 04, 2020.

[27] Sean Boynton, "California wildfire burns 10,000 acres in 3 hours, hundreds of homes evacuated," Global News, California, Aug. 13, 2020.

[28] MARTIN, "Mayon volcano (Luzon Island, Philippines): Volcanic Alert Level lowered to 1," volcanodiscovery.com, Philippines, Jul. 17, 2020.

[29] H. HU, H. ZHOU, J. LI, K. LI and B. PAN, "Automatic and Intelligent Line Inspection using UAV based on Beidou Navigation System," 2019 6th International Conference on Information Science and Control Engineering (ICISCE), Shanghai, China, 2019, pp. 1004-1008.

[30] T. Sugimoto, K. Sugimoto, I. Uechi, N. Utagawa and C. Kuroda, "Efficiency Improvement of Outer Wall Inspection by Noncontact Acoustic Inspection Method using Sound Source Mounted Type UAV," 2019 IEEE International Ultrasonics Symposium (IUS), Glasgow, United Kingdom, 2019, pp. 2091-2094.

[31] K. Asa, Y. Funabora, S. Doki and K. Doki, "Evaluation in Real World of the Measuring Position Determination for Visual Inspection using UAV," IECON 2018 - 44th Annual Conference of the IEEE Industrial Electronics Society, Washington, DC, 2018, pp. 2711-2716.

[32] F. Ge, K. Li, W. Xu and Y. Wang, "Path Planning of UAV for Oilfield Inspection Based on Improved Grey Wolf Optimization Algorithm," 2019 Chinese Control And Decision Conference (CCDC), Nanchang, China, 2019, pp. 3666-3671.

[33] Q. Wang, H. Xiong and B. Qiu, "The Attitude Control of Transmission Line Fault Inspection UAV Based on ADRC," 2017 International Conference on Industrial Informatics - Computing Technology, Intelligent Technology, Industrial Information Integration (ICIICII), Wuhan, 2017, pp. 186-189.

[34] H. Yu, W. Yang, H. Zhang and W. He, "A UAV-based crack inspection system for concrete bridge monitoring," 2017 IEEE International Geoscience and Remote Sensing Symposium (IGARSS), Fort Worth, TX, 2017, pp. 3305-3308.

[35] A. Tariq, S. M. Osama and A. Gillani, "Development of a Low Cost and Light Weight UAV for Photogrammetry and Precision Land Mapping Using Aerial Imagery," 2016 International Conference on Frontiers of Information Technology (FIT), Islamabad, 2016, pp. 360-364.

[36] Zhe Li and $\mathrm{Yan} \mathrm{Li}$, "The best way to promote efficiency and precision of laser scanning for ancient architectures mapping-An ideal rotary-UAV with minimum vibration for LIDAR," 2011 International Conference on Remote Sensing, Environment and Transportation Engineering, Nanjing, 2011, pp. 1761-1766.

[37] M. Hooper et al., "Securing commercial WiFi-based UAVs from common security attacks," MILCOM 2016 - 2016 IEEE Military Communications Conference, Baltimore, MD, 2016, pp. 1213-1218.

[38] K. Yoon, D. Park, Y. Yim, K. Kim, S. K. Yang and M. Robinson, "Security Authentication System Using Encrypted Channel on UAV Network," 2017 First IEEE International Conference on Robotic Computing (IRC), Taichung, 2017, pp. 393-398.

[39] R. Reshma, T. Ramesh and P. Sathishkumar, "Security situational aware intelligent road traffic monitoring using UAVs," 2016 International Conference on VLSI Systems, Architectures, Technology and Applications (VLSI-SATA), Bangalore, 2016, pp. 1-6.

[40] M. Elloumi, R. Dhaou, B. Escrig, H. Idoudi and L. A. Saidane, "Monitoring road traffic with a UAV-based system," 2018 IEEE Wireless Communications and Networking Conference (WCNC), Barcelona, 2018, pp. 1-6.

[41] B. Peng, Y. Li, L. He, K. Fan and L. Tong, "Road Segmentation of UAV RS Image Using Adversarial Network with Multi-Scale Context Aggregation," IGARSS 2018 - 2018 IEEE International Geoscience and Remote Sensing Symposium, Valencia, 2018, pp. 6935-6938.

[42] L. Ichim and D. Popescu, "Road Detection and Segmentation from Aerial Images Using a CNN Based System," 201841 st International Conference on Telecommunications and Signal Processing (TSP), Athens, 2018, pp. $1-5$.

[43] M. F. Ozkan, L. R. G. Carrillo and S. A. King, "Rescue Boat Path Planning in Flooded Urban Environments," 2019 IEEE International Symposium on Measurement and Control in Robotics (ISMCR), Houston, TX, USA, 2019, pp. B2-2-1-B2-2-9.

[44] A. L. Sumalan, D. Popescu, and L. Ichim, "Flood evaluation in critical areas by UAV surveillance," in 8th International Conference on
Electronics, Computers and Artificial Intelligence, ECAI 2016, 2016, pp. 3-8, doi: 10.1109/ECAI.2016.7861118.

[45] T. E. A. Barton and M. A. Hannan Bin Azhar, "Forensic analysis of popular UAV systems," 2017 Seventh International Conference on Emerging Security Technologies (EST), Canterbury, 2017, pp. 91-96.

[46] U. Jain, M. Rogers and E. T. Matson, "Drone forensic framework: Sensor and data identification and verification," 2017 IEEE Sensors Applications Symposium (SAS), Glassboro, NJ, 2017, pp. 1-6.

[47] C. A. Rokhmana and R. Andaru, "Utilizing UAV-based mapping in post disaster volcano eruption," in 2016 6th International Annual Engineering Seminar, InAES, 201AD, pp. 202-205, doi: 10.1109/INAES.2016.7821934.

[48] D. Câmara, "Cavalry to the rescue: Drones fleet to help rescuers operations over disasters scenarios," 2014 IEEE Conference on Antenna Measurements \& Applications (CAMA), Antibes Juan-les-Pins, 2014, pp. 1-4, doi: 10.1109/CAMA.2014.7003421.

[49] E. T. Alotaibi, S. S. Alqefari and A. Koubaa, "LSAR: Multi-UAV Collaboration for Search and Rescue Missions," in IEEE Access, vol. 7, pp. 55817-55832, 2019, doi: 10.1109/ACCESS.2019.2912306.

[50] Te-Chih Liu and Henrik Schmidt, "A framework of real-time seabottom target detection using acoustic sensors on AUVs," Oceans '04 MTS/IEEE Techno-Ocean '04 (IEEE Cat. No.04CH37600), Kobe, 2004, pp. 20192023 Vol.4.

[51] Xiaohu Lv and Y. Liu, "Design of human motion detection and tracking robot based on pyroelectric infrared sensor," 2010 8th World Congress on Intelligent Control and Automation, Jinan, 2010, pp. 3737-3740.

[52] Y. He, F. Le Chevalier and A. G. Yarovoy, "Range-Doppler processing for indoor human tracking by multistatic ultra-wideband radar," 2012 13th International Radar Symposium, Warsaw, 2012, pp. 250-253.

[53] B. Jagadeesh and C. M. Patil, "Video based action detection and recognition human using optical flow and SVM classifier," 2016 IEEE International Conference on Recent Trends in Electronics, Information \& Communication Technology (RTEICT), Bangalore, 2016, pp. 1761-1765.

[54] N. H. Abdul Aziz, M. A. Aminudin, N. H. Abd Rahman and H. Yon, "4G LTE Detection using NEMO Handy for PFSR Support System," 2019 International Symposium on Antennas and Propagation (ISAP), Xi'an, China, 2019, pp. 1-4.

[55] J. Whelan, A. Almehmadi, J. Braverman and K. El-Khatib, "Threat Analysis of a Long Range Autonomous Unmanned Aerial System," 2020 International Conference on Computing and Information Technology (ICCIT-1441), Tabuk, Saudi Arabia, 2020, pp. 1-5, doi: 10.1109/ICCIT144147971.2020.9213789.

[56] M. Tzelepi and A. Tefas, "Human crowd detection for drone flight safety using convolutional neural networks," 2017 25th European Signal Processing Conference (EUSIPCO), Kos, 2017, pp. 743-747, doi: 10.23919/EUSIPCO.2017.8081306.

[57] A. Valsan, P. B., V. D. G. H., R. S. Unnikrishnan, P. K. Reddy and V. A., "Unmanned Aerial Vehicle for Search and Rescue Mission," 2020 4th International Conference on Trends in Electronics and Informatics (ICOEI)(48184), Tirunelveli, India, 2020, pp. 684-687, doi: 10.1109/ICOEI48184.2020.9143062.

[58] J. Yeom, Y. Han, A. Chang and J. Jung, "Hurricane Building Damage Assessment using Post-Disaster UAV Data," IGARSS 2019 - 2019 IEEE International Geoscience and Remote Sensing Symposium, Yokohama, Japan, 2019, pp. 9867-9870, doi: 10.1109/IGARSS.2019.8900477.

[59] Pudasaini, Uttam. (2019). Drones in Disaster Management and Humanitarian Action: A Case Study in The Aftermath of Nepal Earthquake 2015. International Journal of Human and Health Sciences (IJHHS). 19. 10.31344/ijhhs.v0i0.137.

[60] Wesley M. DeBusk, "Unmanned Aerial Vehicle System for Disaster Relief : Tornado Alley", Georgia Institute of Technology, Atlanta, GA, 2019.

[61] National Unmanned Aircraft System Project Office. "Rapid Response : VolcanoMonitoring" https://uas.usgs.gov/nupo/mission/HI_KilaueaVolcano.shtml.

[62] Z. Dukowitz, "Drones Used for First Time Ever by L.A. Fire Department", UAV Coac, 2017.

[63] A. Krizhevsky, I. Sutskever, and G. E. Hinton, "Imagenet classification with deep convolutional neural networks," in Advances in neural information processing systems, 2012, pp. 1097-1105.

[64] C. Szegedy, W. Liu, Y. Jia, P. Sermanet, S. Reed, D. Anguelov, D. Erhan, V. Vanhoucke, and A. Rabinovich, "Going deeper with convolutions," in Proceedings of the IEEE Conference on Computer Vision and Pattern Recognition, 2015, pp. 1-9. 
[65] Y. Taigman, M. Yang, M. Ranzato, and L. Wolf, "Deepface: Closing the gap to human-level performance in face verification," in Proceedings of the IEEE Conference on Computer Vision and Pattern Recognition. IEEE, 2014, pp. 1701-1708

[66] D. Ciresan, U. Meier, and J. Schmidhuber, "Multi-column deep neural networks for image classification," in Computer Vision and Pattern Recognition (CVPR), 2012 IEEE Conference on. IEEE, 2012, pp. 36423649.

[67] Y. LeCun, L. Jackel, L. Bottou, C. Cortes, J. S. Denker, H. Drucker, I. Guyon, U. Muller, E. Sackinger, P. Simard et al., "Learning algorithms for classification: A comparison on handwritten digit recognition," Neural networks: the statistical mechanics perspective, vol. 261, p. 276, 1995.

[68] A. Toshev and C. Szegedy, "Deeppose: Human pose estimation via deep neural networks," in Proceedings of the IEEE Conference on Computer Vision and Pattern Recognition, 2014, pp. 1653-1660.

[69] R. Girshick, J. Donahue, T. Darrell, and J. Malik, "Rich feature hierarchies for accurate object detection and semantic segmentation," in Proceedings of the IEEE conference on computer vision and pattern recognition, 2014, pp. 580-587.

[70] P. Sermanet, K. Kavukcuoglu, S. Chintala, and Y. LeCun, "Pedestrian detection with unsupervised multi-stage feature learning," in Computer Vision and Pattern Recognition (CVPR), 2013 IEEE Conference on. IEEE, 2013, pp. 3626-3633.

[71] A. Babenko, A. Slesarev, A. Chigorin, and V. Lempitsky, "Neural codes for image retrieval," in Computer Vision-ECCV 2014. Springer, 2014. pp. 584-599.

[72] J. Wan, D. Wang, S. C. H. Hoi, P. Wu, J. Zhu, Y. Zhang, and J. Li, "Deep learning for content-based image retrieval: A comprehensive study," in Proceedings of the ACM International Conference on Multimedia. ACM, 2014, pp. 157-166.

[73] Y. Naidoo, R. Stopforth and G. Bright, "Development of an UAV for search \& rescue applications," IEEE Africon '11, Livingstone, 2011, pp. $1-6$

[74] S. Waharte and N. Trigoni, "Supporting Search and Rescue Operations with UAVs," 2010 International Conference on Emerging Security Technologies, Canterbury, 2010, pp. 142-147.

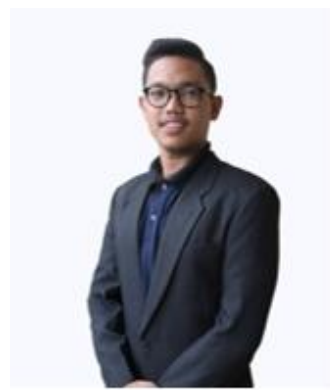

Muhammad Shahir Hakimy Bin Salem was born in Johor, Malaysia, in 1995. He obtained his B. Eng. (Hons) Electronics Engineering from Universiti Teknologi MARA (UiTM), Shah Alam, Malaysia, in 2019. He obtained his Diploma in Electrical Engineering (Electronics) from Universiti Teknologi MARA (UiTM), Dungun, Malaysia, in 2016. He is pursuing a Master of Science in the Faculty of Electrical Engineering, Universiti Teknologi MARA (UiTM), Shah Alam, Malaysia. His current research interests include human detection and localization for drone application at the disaster area.

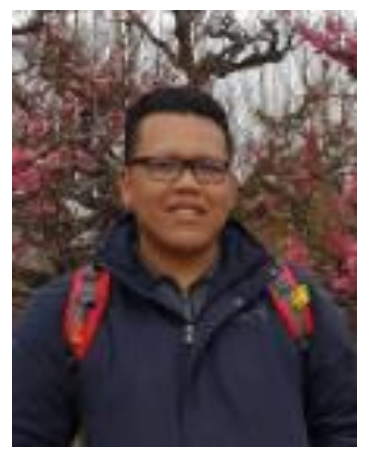

Fadhlan Hafizhelmi Kamaru Zaman received the B.Sc. (Hons.) and Ph.D. degrees from International Islamic University Malaysia in 2008 and 2015, respectively. He is currently the Head of Vehicle Intelligence and Telematics Lab, Universiti Teknologi MARA, Malaysia. His research interests are in pattern recognition, signal and image processing, artificial intelligence, and computer vision. Fadhlan is a Professional Engineer with Board of Engineer Malaysia (BEM) member of IEEE, Professional Technologist with Malaysian Board of Technologist (MBOT), and a Chartered Engineer from the Institution of Engineering and Technology, UK. He has published more than 50 research articles on pattern recognition, signal and image processing, artificial intelligence, and computer vision.

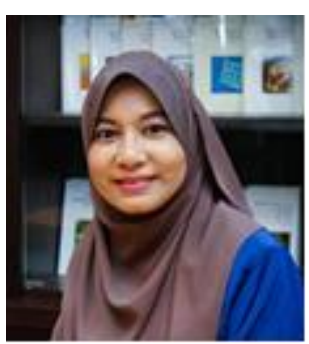

Nooritawati Md Tahir is a lecturer at the Faculty of Electrical Engineering, Universiti Teknologi MARA (UiTM), Selangor, Malaysia. Her research interest is in the field of Image Processing, Pattern Recognition and Computational Intelligence. She has authored and coauthored more than 250 indexed publications with SCOPUS h-index of 18. In addition, she is a Certified Trainer for Postgraduate Training in Innovation and Entrepreneurship awarded by TCD-UCD Dublin, Ireland, Stanford Faculty Fellow organised by Stanford Technology Ventures Program, Stanford University, US and Chartered Engineer (CEng) from the Institution of Engineering and Technology and Engineering Council UK as well as Senior Member (SMIEEE) of Institution of Electrical, Electronic Engineer (IEEE). 